\title{
THE NOVEMBER MEETING IN CHICAGO
}

The four hundred fifty-first meeting of the American Mathematical Society was held at the Illinois Institute of Technology on Friday and Saturday, November 25-26, 1949.

The total attendance was about 175 including the following 151 members of the Society:

A. A. Albert, W. R. Allen, W. F. Atchison, W. L. Ayres, Reinhold Baer, R. G. Bartle, S. F. Bibb, K. E. Bisshopp, L. M. Blumenthal, D. G. Bourgin, A. J. Brandt, Richard Brauer, R. H. Bruck, J. R. Buchi, J. W. Butler, S. S. Cairns, A. P. Calderon, R. H. Cameron, C. S. Carlson, S. S. Chern, Herman Chernoff, H. M. Clark, E. H. Clarke, M. D. Clement, Harvey Cohn, B. H. Colvin, E. G. H. Comfort, J. J. Corliss A. R. Craw, H. J. Curtis, M. M. Day, John DeCicco, R. F. Deniston, D. M. DeWitt, Flora Dinkines, N. J. Divinsky, J. M. Dobbie, R. J. Duffin, John Dyer-Bennet, D. O. Ellis, R. L. Erickson, M. H. M. Esser, H. P. Evans, R. L. Evans, D. A. Flanders, L. R. Ford, Abraham Franck, Evelyn Frank, D. M. Friedlen, C. G. Fry, R. E. Fullerton, Albert Furman, J. W. Gaddum, Abe Gelbart, D. G. Gore, S. H. Gould, L. M. Graves, Harold Greenspan, M. M. Gutterman, P. R. Halmos, Charles Hatfield, E. D. Hellinger, I. N. Herstein, G. P. Hochschild, C. C. Hsiung, H. K. Hughes, Herbert Jehle, Ralph Hull, W. E. Jenner, M. M. Johnsen, L. H. Kanter, Irving Kaplansky, L. M. Kelly, D. E. Kibbey, Jacob Korevaar, W. C. Krathwohl, M. Z. Krzywoblocki, H. G. Landau, E. P. Lane, R. E. Langer, K. B. Leisenring, Howard Levin, D. J. Lewis, Saunders MacLane, H. M. MacNeille, W. H. Marlow, M. S. Matchett, C. W. Mathews, A. E. May, Karl Menger, J. M. Mitchell, J. T. Moore, C. W. Moran, K. A. Morgan, D. R. Morrison, Leopoldo Nachbin, M. J. Norris, Daniel Orloff, E. H. Ostrow, Gordon Pall, M. C. Peixoto, M. M. Peixoto, Sam Perlis, D. H. Potts, G. B. Price, L. E. Pursell, A. L. Putnam, W. P. Reid, W. T. Reid, Haim Reingold, L. G. Riggs, A. E. Ross, J. M. Sachs, R. G. Sanger, A. C. Schaeffer, K. C. Schraut, W. T. Scott, I. E. Segal, B. R. Seth, S. S. Shü, J. H. Siedband, G. F. Simmons, I. M. Singer, M. L. Slater, D. M. Smiley, M. F. Smiley, Albert Soglin, Daniel Sokolowsky, E. H. Spanier, E. J. Specht, C. E. Springer, Marvin Stippes, E. B. Stouffer, A. F. Strehler, T. T. Tanimoto, C. J. Thomas, E. F. Trombley, H. L. Turrittin, J. L. Ullman, F. A. Valentine, Bethuhne Vanderburg, Robert Wasserman, André Weil, George Whaples, L. R. Wilcox, A. W. Wundheiler, J. E. Yarnelle, J. W. T. Youngs, R. K. Zeigler, Daniel Zelinsky, Antoni Zygmund.

By invitation of the Committee to Select Hour Speakers for Western Sectional Meetings, Professor R. H. Bruck delivered an address entitled $A n$ extension theory for a certain class of loops.

Sessions for the presentation of contributed papers were held at 10:30 A.M. on Friday and Saturday and at 3:15 P.M. on Friday.

Presiding officers for the various sessions were Professors Reinhold Baer, L. M. Blumenthal, L. R. Ford, Gordon Pall, and Dr. H. M. MacNeille.

On Friday afternoon there was a tea for the Society in the Metallurgical and Chemical Engineering Building. 
Abstracts of papers read follow below. Papers read by title are indicated by the letter " $t$." Paper number 46 was read by Mr. Calderon and paper number 51 by Dr. Slater.

\title{
Algebra ANd Theory of Numbers
}

\author{
39. Richard Brauer: On the zeta functions of algebraic number \\ fields. II.
}

Consider algebraic number fields $K$ of degree $n$ over the field of rational numbers and denote the discriminant by $d$, the class number by $h$, and the regulator by $R$. In an earlier paper (Amer. J. Math. vol. 69 (1947) pp. 243-250) the asymptotic relation $\log (h R) \sim \log |d|^{1 / 2}$ was proved for fields of a fixed degree $n$. This result is now extended to sequences of fields normal over the field of rational numbers for which $\log |d| / n \log n \rightarrow \infty$ (where $n$ is not fixed). A weaker result is obtained for fields which are not normal. (Received October 13, 1949.)

\section{M. M. Day: Amenable groups. Preliminary report.}

The definitions of amenable and strongly amenable groups were given in Bull. Amer. Math. Soc. Abstract 55-11-507. Theorem 1. If $G$ is (strongly) amenable, so is every subgroup of $G$ and every factor group of $G$. Theorem 2. $G$ is (strongly) amenable if and only if every finitely generated subgroup of $G$ is (strongly) amenable. Corollary. No amenable group has a free subgroup on more than one generator. (Received October 14, 1949.)

\section{D. O. Ellis: Autometrized Boolean algebras.}

Let $B$ be a Boolean algebra with meet, join, and complement denoted by $a b, a+b$, and $a^{\prime}$ respectively. Defining "distance" of $a$ and $b$ by $d(a, b)=a^{\prime} b+a b^{\prime}$, we call such a system an autometrized Boolean algebra. The notions of betweenness, congruence, superposability, and free mobility are defined in terms of this "distance" analogously to the definitions of these concepts in a metric space. It is shown that betweenness is equivalent to general lattice betweenness (Pitcher and Smiley, Trans. Amer. Math. Soc. vol. 52 (1942) pp. 95-114), and that $B$ has the property of free mobility. A $B$ metrized space is any abstract set with symmetric "distances" in $B$ which satisfy the vanishing condition. It is shown that if each three points of an arbitrary $B$-metrized space are congruently contained in $B$ then the space is congruently contained in $B$, and that three is the smallest number with that property. (Received September 30, 1949.)

42t. Marshall Hall: $A$ basis for free Lie rings and higher commutators in free groups.

E. Witt has found the number $\psi n(q)$ of linearly indeprendent elements of degree $n$ in the free Lie ring with $q$ generators but his method gives no way of finding a basis. By an isomorphism, $\psi n(q)$ is also the number of independent commutators of weight $n$ modulo weight $n+1$ in the free group with $q$ generators. An explicit construction is given in this paper for a basis of the Lie ring and the corresponding higher commutator groups. The commutators are precisely those which arise in Philip Hall's collecting process as given in $A$ contribution to the theory of groups of prime power order, Proc. London Math. Soc. vol. 36 (1934) pp. 29-95, (Received September 25, 1949.) 


\section{3t. Irving Kaplansky: Topological representation of algebras. II.}

The previous paper by Arens and the author was devoted mainly to the study of commutative algebraic algebras. In the present paper commutativity is weakened to the assumption of a polynomial identity. Such algebras are shown to be built out of certain uniquely determined homogeneous parts, and the latter are determined if a countability assumption is satisfied. There are applications to Kurosch's problem of determining whether algebraic algebras are locally finite: (1) the answer is affirmative for the case of a polynomial identity, (2) if the answer is affirmative for primitive algebras and nil algebras, then it is affirmative for all algebraic algebras. (Received September 20, 1949.)

\section{4t. B. E. Meserve: A note on polynomial equations and inequalities.}

Methods for the determination of the number $r$ of $n$-tuples of real numbers satisfying the system (A) $0<f_{i}(i=1,2, \cdots, p), 0=f_{i}(i=p+1, \cdots, m)$ where the $f_{i}$ are polynomials in $x_{1}, x_{2}, \cdots, x_{n}$ with real coefficients have been given by A. Tarski ( $A$ decision method for elementary algebra and geometry, Report 109, Project Rand) when $r$ is finite. If $p=m$, the resulting system (B) $0<f_{i}(i=1,2, \cdots, m)$ is either inconsistent or $r$ is infinite. In the latter case the system is satisfied for $n$-tuples lying in a certain number $k$ of $n$-dimensional maximal open segments. These segments form the content of the system (B). The determination of $k$ constitutes an extension of Sturm's Theorem to polynomial inequalities and has not in general been solved. Solutions have been given by the writer for the case of one unknown (Amer. J. Math. (1947) pp. 357-370) and, in this paper, for the system (C) $0<b_{i} E_{i}(i=1,2, \cdots, n)$ where $E_{i}$ is the elementary symmetric polynomial of degree $i$ in $x_{1}, x_{2}, \cdots, x_{n}$. The system (C) is consistent if and only if $0 \neq b_{1} b_{2} \cdots b_{n}$ and if consistent has $k=n ! /[V !(n-V) !]$ where $V$ is the number of variations of sign in the sequence $1, b_{1}, b_{2}, \cdots, b_{n}$. Using extensions of Descartes' rule of signs the content of (C) is explicitly given for all cases in which $k=1$ or $n$. (Received October 11, 1949.)

45. Gordon Pall: Simultaneous representation by adjoint quadratic forms.

An algorithm is given for constructing all simultaneous solutions of $a$ $=f\left(x_{1}, \cdots, x_{n}\right), 0=x_{1} y_{1}+\cdots+x_{n} y_{n}, b=g\left(y_{1}, \cdots, y_{n}\right)$, in integers $x_{i}, y_{j}$ such that $\left(x_{1}, \cdots, x_{n}\right)=\left(y_{1}, \cdots, y_{n}\right)=1$. Here $f$ is any real nonsingular quadratic form, and $g$ is its adjoint form. In general, there is a precise formula connecting the number of "sets" of solutions by the system of classes of a genus, and certain classes of quadratic forms in $n-2$ variables along with the numbers of solutions of certain systems of quadratic congruences. The results are especially simple if $f$ is in a genus of one class, and if $a$ and $b$ are relatively prime integers. Thus, to state the simplest example, if $a$ and $b$ are positive and relatively prime, the number of solutions of $a=x_{1}^{2}+x_{2}^{2}+x_{3}^{2}$, $0=x_{1} y_{1}+x_{2} y_{2}+x_{3} y_{2}, b=y_{1}^{2}+y_{2}^{2}+y_{3}^{2}$, in integers such that $x_{1}, x_{2}, x_{3}$ are relatively prime and $y_{1}, y_{2}, y_{3}$ are relatively prime, is equal to $24 k k^{\prime}$, where $k$ denotes the number of solutions of $t^{2} \equiv-b \bmod a$, and $k^{\prime}$ is the number of solutions of $u^{2} \equiv-a \bmod b$, (Received October 11, 1949.)

\section{ANALYsis}

46. A. P. Calderon and Antoni Zygmund: Note on the boundary values of functions of several complex variables. 
(i) Let $f\left(z_{1}, \cdots, z_{k}\right)$ be a function regular in the polycylinder $\left|z_{1}\right|<1, \cdots,\left|z_{k}\right|<1$, and satisfying the condition $\int_{0}^{2 \pi} \cdots \int_{0}^{2 \pi} \log +|f| d \theta_{1} \cdots d \theta_{k}=O(1)$, where $f$ stands for $f\left(r_{1} e^{i \theta_{1}}, \cdots, r_{k} e^{i \theta_{k}}\right)$. Then $f$ has a finite limit as $\left(z_{1}, \cdots, z_{k}\right)$ tends nontangentially to almost every point $\left(e^{i \theta_{1}^{0}}, \cdots, e^{i \theta^{0}}\right)$, provided all the ratios $\left(1-r_{j}\right) /\left(1-r_{h}\right)$ are contained between two positive numbers. (The nontangential approach just quoted means that each $z_{j}$ tends nontangentially to $e^{i \theta^{0}}$.) The proof of (i) is based on the following theorem. (ii) Let $f\left(z_{1}, \cdots, z_{k}\right)$ be regular for $\left|z_{1}\right|<1, \cdots,\left|z_{k}\right|<1$, and suppose a set $E$ situated on the distinguished boundary $\left(D_{k}\right) z_{1}=e^{i \theta_{1}}, \cdots, z_{k}=e^{i \theta_{k}}$ of the unit polycylinder has the following property: as $\left(z_{1}, \cdots, z_{k}\right)$ approaches any point $\left(e^{i \theta_{1}^{0}}, \ldots, e^{i \theta_{k}^{0}}\right) \in D_{k}$ nontangentially and so that all the ratios $\left(1-r_{j}\right) /\left(1-r_{h}\right)$ are contained between two posiive numbers, the function $f$ remains bounded. Then, under the same circumstances, the function $f$ tends to a finite limit for almost every point $\left(e^{i \theta_{1}^{0}}, \cdots, e^{i \theta}{ }^{0}\right) \in E$. (Received October 13, 1949.)

\section{R. H. Cameron: The first variation of an indefinite Wiener inte-} gral.

It is shown in this paper that if $G(u)$ is the Wiener integral of the functional $F(x)$ over the set $S(u)$ of continuous functions $x(t)$ on $0 \leqq t \leqq 1$ which vanish at $t=0$ and satisfy for all $t$ the inequality $x(t) \leqq u(t)$, then under suitable smoothness conditions on $F(x)$ and $u(t)$ and $\delta u(t)$ we have $\delta G(u)$ equal to the Wiener integral over $S(u)$ of the expression $\delta F(x)-2 F(x) \int_{0}^{1} \delta u^{\prime}(t) d x(t)$. Here it is understood that the variation $\delta F(x)$ is to be based on the independent variation $\delta x(t)$ when the latter is put equal to $\delta u(t)$. (Received October 13, 1949.)

48. R. L. Evans: Asymptotic solutions of some linear ordinary differential equations containing a parameter. Preliminary report.

A method of solution used earlier by R. E. Langer (Trans. Amer. Math. Soc. vol. 36 (1934) pp. 90-106) is generalized. Asymptotic solutions with error terms of the order $\left(1 / \lambda^{p+k_{0}}\right)^{1-\epsilon}$ have been found for the equations $y^{\prime \prime}-\lambda^{2 p}\left(\sum_{j=1}^{m}\left(\lambda^{p} x\right)^{i} \cdot \phi_{p j}\right.$ $\left.+\sum_{j=1-2 p-k_{0}}^{J} \lambda^{i} \cdot \phi_{j}+\psi_{k_{0}}(\lambda, x)\right) y=0$ in which the indices are integers such that $m=1$, $p=2, J=1$, and $k_{0} \geqq 0$ or that $m=2, p=1$, and $J=k_{0}=0$, in which latter case a specific relation must exist among the coefficients of the $\phi$ 's. The $\phi$ 's are analytic functions of $x$, $\boldsymbol{\phi}_{m p}(0) \neq 0$, and $\psi_{k_{0}}$ is such that $\lambda^{2 p+k_{0}} \cdot \psi_{k_{0}}$ is an analytic function of $x$ for sufficiently small $|x|$ and bounded for all sufficiently large $|\lambda|$. The method can be extended to certain equations in which the order is larger and/or the indices $m$ and $p$ have values other than 1 and 2. As $m$ is increased the number of supplementary conditions on the $\phi$ coefficients is also increased. (Received October 22, 1949.)

\section{Abraham Franck: On analytic functions of bounded type.}

Extending results of A. Wishard (Duke Math. J. vol. 9 (1942) p. 663) the author obtains criteria for an analytic function to be of bounded type (B.T.) in $\Re(z)=x>0$ : $f(z)$ is of B.T. in $x>0$ if and only if there exists either (i) a sequence of circles $C_{n}$ which exhausts $x>0$ such that $\iint_{c_{n}}\left(\log +|f(z)| /|1+z|^{2}\right) d x d y \leqq M R_{n}, M=$ const., $R_{n}$ radius of $C_{n}$; or (ii) a sequence of circles $C_{n}^{*}$ center on $x$-axis passing through $x=\rho_{n}$, $x=1 / \rho_{n}, \lim \rho_{n}=\infty$ such that $\iint_{C_{n}^{*}}\left(\log +|f(z)| /|1+z|^{2}(d x d y / x) \leqq M \log \rho_{n}\right.$. By means of these results, extensions of criteria of A. Ostrowski and R. Nevanlinna are obtained for an analytic function to be of B.T. in a simply-connected region $R$ possessing the following property: if $w=\phi(z)$ maps $R$ conformally onto $|w|<1$, two positive constants $c_{1}$ and $c_{2}$ exist such that $c_{1} \leqq\left|\phi^{\prime}(z)\right| \leqq C_{2}$. The analytic function $f(z)$ is of B.T. 
in $R$ if and only if there exists either (i) some sequence of regions $D_{n}$ which exhausts $R$ and a point $z=a$ on the boundary of $R$ such that $\iint_{D_{n}}\left(\log +|f(z)| /|a-z|^{2}\right) d x d y$ $\leqq M / d_{n}, d_{n}$ the Fréchet distance of the boundary of $D_{n}$ to the boundary of $R$; or (ii) some sequence of regions $D_{n}^{*}$ which exhausts $R$ such that $\left.\iint_{D_{n}^{*}(\log }+|f(z)| / d_{n}(z)\right) d x d y$ $\leqq M / d_{n}, d_{n}(z)$ is the distance of the point $z$ from the boundary of $R$. Several applications are given. (Received October 13, 1949.)

\section{Jacob Korevaar. A theorem on power series with partial sums} which are free of zeros in a sector.

If no partial sum of a formal power series (1) $\sum a_{n} z^{n}$ has a zero in the sector $|\arg z|$ $\leqq \alpha(\alpha>0)$, then (1) represents an entire function of order zero. This theorem extends and improves results of L. Weisner for $\alpha>\pi / 2$ (Bull. Amer. Math. Soc. vol. 47 (1941) pp. 160-163) and of O. Szász for $\alpha=\pi / 2$ (Bull. Amer. Math. Soc. vol. 49 (1943) pp. $377-383$, Theorem II). It is first proved that (1) converges for some $z \neq 0$. Suppose that on the contrary lim $\sup \left(C_{k}=\left|a_{k}\right| 1 / k\right)=\infty$. For the values of $n$ for which $C_{n} \geqq C_{k}(k<n)$, $f_{n}(t)=\left(1+\cdots+a_{n-p} a_{n}^{-1} C_{n}^{p} t^{p}+\cdots+a_{0} a_{n}^{-1} C_{n}^{n} t^{n}\right)^{1 / n} \rightarrow 1$, boundedly and hence uniformly in $|\arg t| \leqq \alpha-\eta, \delta<|t|<d(\eta, \delta, d>0)$. In particular $\mid\left\{f_{n}(t)\right\}^{n}<2^{n}\left(n>n_{0}\right)$ on the interval $1 \leqq t \leqq 10$ of length 9 . Hence by Tchebycheff's inequality inf $\left|g^{(n)}(t)\right|$ $\leqq 2^{-1} n !\{4 /(b-a)\}^{n}$ sup $|g(t)|$, applied to $g(t)=\operatorname{Re}\left\{f_{n}(t)\right\}^{n}$ and $\operatorname{Im}\left\{f_{n}(t)\right\}^{n}, a=1$, $b=10, a_{0}$ must be zero, which is absurd. But if (1) converges for some $z \neq 0, \mathrm{R}$. Jentzsch's theorem on the zeros of partial sums implies that (1) converges everywhere. Finally, F. Carlson's results on the zeros of the partial sums of entire functions of positive or infinite order (Arkiv. för Matematik, Astronomi och Fysik vol. 35A (1948) no. 14) imply that (1) represents an entire function of order zero. The theorem remains true if for every $n$ there is a sector $\left|\arg z-\phi_{n}\right| \leqq \alpha(\alpha>0$ independent of $n)$ in which $\sum_{k \leqq n} a_{k} z^{k}$ has no zero. (Received October 13, 1949.)

51. M. J. Norris, M. L. Slater, and C. J. Thomas: Functional inequalities suggested by game theory. Preliminary report.

The main results are: 1 . If (i) $f(x)$ is nondecreasing and continuous in $(-\infty, \infty)$, (ii) $p(t)$ is nondecreasing in $[0,1]$, (iii) $\alpha(t) \in L(0,1)$, (iv) $\int_{0}^{1} \alpha d t=k$, and $F(x)$ denotes $\int_{0}^{x} f(x) d x$, then $\int_{0}^{1} \alpha f\left(\int_{0}^{t} \alpha p d t\right) d t \geqq F[p(0+) k] / p(0+)$ when $p(0+) \neq 0$ and $\geqq k f(0)$ when $p(0+)=0$. 2. If (i) $f(x)$ is continuous and $\int_{0}^{x} f d x / x$ is nondecreasing in $(-\infty, \infty)$, (ii) $p(t)$ is nondecreasing in $[0,1]$ and $\geqq 0$, (iii) $\alpha(t) \in L(0,1)$, (iv) $\int_{0}^{1} \alpha d t=0$, then $\int_{0}^{1} \alpha f\left(\int_{0}^{t} \alpha p d t\right) d t \geqq 0$. 3. If (i) $f(x, y)$ is continuous in the $(x, y)$-plane (ii) $f(x+h, y+k)$ $-f(x+h, y)-f(x, y+k)+f(x, y) \geqq 0$ for all $x, y, h, k$, (iii) $p(s)$ nondecreasing in $[0,1]$ and $q(t)$ nondecreasing in $[0,1]$, (iv) $\alpha(s) \in L(0,1), \beta(t) \in L(0,1),(\mathrm{v}) \int_{0}^{1} \alpha d s=\int_{0}^{1} \beta d t$ $=0$, then $\int_{0}^{1} \int_{0}^{1} \alpha(s) \beta(t) f\left(\int_{0}^{\alpha} \alpha p d s, \int_{0}^{t} \beta q d t\right) d s d t \geqq 0$. Continuity restrictions on $f(x)$ and $f(x, y)$ can in general be weakened, but with a loss in compactness in stating the final results. Various partial converses have been proved. (Received November 2, 1949.)

\section{W. T. Scott: The reciprocal of a continued fraction.}

In this note it is shown that the power series-continued fraction correspondence $P(x) \equiv 1+\sum c_{n} x^{n} \sim 1+K\left(a_{n} x^{\alpha_{n}} / 1\right)$, where $\alpha_{2 n-1}=\alpha_{2 n}$, a positive integer, implies the correspondence $1 / P(x) \sim 1+K\left(b_{n} x^{\alpha_{n}} / 1\right)$ if and only if there exist parameters $h_{p}$ such that $a_{1}=2 h_{1}, a_{2 n}=h_{2 n-1}\left(h_{2 n}-1\right), a_{2 n+1}=\left(h_{2 n}+1\right) h_{2 n+1}$. In this case the $b_{n}$ are obtained from the $a_{n}$ by replacing each $h_{p}$ by $-h_{p}$. The result, which includes a theorem of Stieltjes, yields several new continued fraction expansions. (Received October 13, 1949.) 


\section{I. E. Segal: An extension of Plancherel's formula to separable unimodular groups.}

Any complex-valued function on a separable unimodular locally compact group which is square-integrable relative to Haar measure has the integral of the square of its absolute value equal to the integral over a certain measure space of the square of the relative norm (at each point of the space) of the Fourier transform of the function. The "relative norm" referred to is that defined by von Neumann for operators in factors, and the Fourier transform is defined through the use of the von Neumann reduction theory, together with a limiting process. The measure space in question has its measure ring isomorphic to the Boolean ring of closed linear manifolds in $L_{2}(G)$ invariant under both left and right translations, and the measure on it is unique modulo the normalization of the relative norms. Use is made of a certain countablyadditive unitarily-invariant non-negative-valued function $w$ defined, on the projections in the weak closure $L$ of the algebra generated by the left regular representation, by the equations $w(P)=\|f\|^{2}$ if $P$ is convolution by the element $f$ of $L_{2}(G)$, and $w(P)$ $=\infty$ otherwise. Any projection in $L$ is the least upper bound of projections in $L$ on which $w$ is finite, and it results that factors of type III do not occur in the reduction of $L$ with respect to its center. (Received October 12,1949.)

54. E. F. Trombley: Extension of M. Riesz's theorem on Hilbert transforms to functions of two variables.

Here the methods of K. Sok61-Sokolowski (Fund. Math. vol. 34 (1947) pp. 170177) are used to show that if $f(x, y)$ is a function in $L_{p}(-\infty<x, y<\infty)(p>1)$, then the Hilbert transform $H f$ of $f$ satisfies a relation of the form $\|H f\|_{p} \leqq K_{p}\|f\|_{p}$ where $K_{p}$ is a function of $p$ alone. In the course of the proof it is shown that: If $f(x)$ is a function in $L_{p}(-\infty<x<\infty)(p>1)$, then the function $\widetilde{f}(x)=\sup _{\sigma>0}\left|\left(\int_{-\infty}^{-\sigma}+\int_{0}^{\infty}\right)(f(x+t) / r) d t\right|$ satisfies the inequality: $\|\widetilde{f}\|_{p} \leqq A_{p}\left\|_{f}\right\|_{p}$ where $A_{p}$ is a function of $p$ alone. This result is extended to functions of two variables and then used to obtain the above result on Hilbert transforms. (Received October 13, 1949.)

\section{J. L. Ullman: A mapping property of schlicht functions.}

The mapping property that is proved in the article holds for the normalized exterior mapping function of a simple analytic curve. Let $C$ be a simple analytic curve in the $z$ plane and designate its exterior by $D$. The normalized exterior mapping function of $C$ is the analytic function $w=f(z)$ which is uniquely determined by conditions that (i) it is regular in $D$ except for a simple pole at $z=\infty$, (ii) its power series expansion about $z=\infty$ has the normalization $w=z+a+b / z+\cdots$; and (iii) it maps $D$ in a 1-1 manner onto the exterior of a circle $\Sigma,|w|=\rho$. Theorem I. Let $C$ be a simple analytic curve, and designate its exterior by $D$. Let $f(z)$ be the normalized exterior mapping function of $C$. Let $\sigma$ be a circle whose interior lies in $D$ and with center at $z_{0}$. Then $F(z)=f(z) /\left(z-z_{0}\right)$ maps $\sigma$ onto a curve in the $w$ plane that is star-shaped from the point $w=0$. If $\sigma$ is a circle containing $C$, then the function $f(z)$ maps $\sigma$ onto a curve in the $w$ plane that is starshaped from the point $w=0$. The first part of this theorem gives part of the domain in which the ratio of two schlicht functions is again schlicht. The second part provides a complement to the result stated in problem 317, section III of Aufgaben und Lehrsätze aus der Analysis by P6lya and Szegö. (Received October 13, 1949.) 
56. Bethuhne Vanderburg: A linear combination of Cesàro and Hölder summability methods. Preliminary report.

Let $H_{\alpha}, C_{\alpha}$ denote the Hölder and Cesàro summability methods of order $\alpha, H_{n}^{\alpha}$, $C_{n}^{\alpha}$ the $n$th Hölder and Cesàro transformations of any sequence $\left\{s_{n}\right\}_{0}^{\infty}$. The following are proven. First, for real values of $\alpha>-1$ excepting 0 and 1 , the summability method $\left[C_{\alpha}-\Gamma(\alpha+1) H_{\alpha}\right][1-\Gamma(\alpha+1)]^{-1}$ is equivalent to the method $H_{\alpha+1}$. Secondly, if $\alpha>-1$ and if $\left\{s_{n}\right\}_{0}^{\infty}$ is limitable- $H_{\alpha+1}$ to $s$, then $\lim \sup _{n \rightarrow \infty}$ (inf) $\left[C_{n}^{\alpha}-s\right]$ $=\lim \sup _{n \rightarrow \infty}\left(\right.$ inf) $\Gamma(\alpha+1)\left[H_{n}^{\alpha}-s\right]$. (Received November 18, 1949.)

\section{Applied Mathematics}

\section{Herbert Jehle: Two component wave equations.}

In the case of vanishing electromagnetic potentials $\phi_{\mathrm{K}}=0$, the relativistic second order Schroedinger-Klein-Gordon equation can be satisfied by iterating the equation $\gamma^{\kappa}\left(\partial / \partial x^{\kappa}-i \phi_{k}\right) \psi=\mu \psi^{*}, \psi^{*}=$ conjugate complex of $\psi, \mu$ is a real constant, where the $\gamma^{\kappa}$ have to satisfy $\left(\gamma^{\kappa *} \gamma^{\lambda}+\gamma^{\lambda *} \gamma^{\kappa}\right) / 2=-g^{\kappa \lambda} 1$ with $g^{00}=+1, g^{11}=g^{22}=g^{33}=-1$. The latter equation has, apart from equivalence transformations, two essentially different types of solutions by $2 \times 2$ matrices, that is, $\gamma^{0}$, the matrix with first row $0,-1$ and second row 1,$0 ; \gamma^{1}$, the matrix with first row $i, 0$ and second row $0, i ; \gamma^{2}$, the matrix with first row 0,1 and second row 1,$0 ; \gamma^{3}$, the matrix with first row 1,0 and second row $0,-1$; and the conjugate complex of these matrices. If the potentials $\phi_{k} \neq 0$, such a Lorentz covariant two-component wave equation is no longer covariant with respect to coordinate reflections. If the first type of solutions $\gamma^{k}$ describes the motion of a positive charge in a given potential $\phi_{k}$, the second type describes that of a negative charge. The equation permits the definition of a real current four vector whose divergence vanishes identically by virtue of the wave equation. Gauge invariance is satisfied, and the superposition principle holds, but with arbitrary real coefficients. The relation of this equation to a real 4 component equation is analyzed by $\mathrm{J}$. Serpe, Physical Review vol. 76 (1949) (in print). (Received October 25, 1949.)

58. M. Z. Krzywoblocki: On the extension of the Kolmogoroff theory of turbulence to compressible fluids.

Kolmogoroff's theory of locally isotropic turbulence proposed originally for an incompressible fluid was extended to flows of a compressible fluid. The following four equations were taken into account: equation of motion, conservation of mass, state, and energy. The first and second similarity hypotheses were extended to cover the domain of compressible fluids. The conclusions derived from the reduced equations of motion and energy show a full agreement with the similarity hypotheses. (Received October 10, 1949.)

\section{B. R. Seth: Finite elasto-plastic rotation of a cylinder.}

In a previous paper it has been shown that, for a first approximation, both the plastic flow and deformation theories give similar results for the torsion of a circular cylinder. A second approximation, obtained by using finite deformation, has revealed that, unlike the plastic flow theory, the deformation theory gives discontinuities in the stresses across the elasto-plastic boundary, and hence cannot be expected to give satisfactory results. This method is now extended to treat the finite elasto-plastic rotation of a circular cylinder. (Received October 7, 1949.) 
60. S. S. Shü: On Taylor-Maccoll's equation. II. An analytic formula of the solution in a series form.

The existence theorem of conical flows with an attached conical shock wave was established mathematically some time ago by the author. The present note is to give an analytic solution of Taylor-Maccoll's equation when the tangential component of the flow velocity at the solid cone is prescribed. Because of the singularity inside the solid cone, a series expansion in terms of the semi-apex angle from the axis of the solid cone in general does not converge in the whole domain concerned. A transformation is performed so that the solution in series form is valid for the whole domain between the solid cone and the shock wave. Explicit formulas up to seven terms are worked out. Results are compared with Kopal's recent refined numerical calculations for the cone at Massachusetts Institute of Technology in excellent agreement. (Received October 13, 1949.)

\section{1t. L. A. Zadeh: An application of Fourier transformation to the} solution of linear differential equations with variable coefficients.

A method developed by the author for the analysis of linear variable networks (Frequency analysis of variable networks, Dissertation, Columbia University, 1949) can be applied with advantage to the solution of a wide class of linear differential equations. Consider $L(p ; t) y(t)=K(p ; t) x(t)$ where $p=d / d t ; L$ and $K$ are linear operators; $x(t)$ is a prescribed function of time $(x(t)=0$ for $t<0)$, and $y(t)$ and all of its derivatives are zero for $t<0$. Define $W(t, \xi)$ as the solution corresponding to $x(t)=\delta(t-\xi)$ (Dirac's delta function). We have $W(t, \xi)=K^{*}(p ; \xi) G(t, \xi)\left(p=d / d \xi, K^{*}=\right.$ adjoint of $\left.K\right)$ where $G(t, \xi)$ is Green's function for $L(p ; t)$ corresponding to the above conditions on $y$. Define the system function of the given equation as $H(j w ; t)=\int_{-\infty}^{\infty} W(t, \xi) e^{-j w(t-\xi)} d \xi$. Define the bi-frequency system function as $\Gamma(j w ; j u)=\int_{-\infty}^{\infty} H(j w ; t) e^{-j u t} d t$. It is shown that $y(t)=(2 \pi)^{-1} \int_{-\infty}^{\infty} H(j w ; t) X(j w) e^{j w t} d w$ and $Y(j w)=\int_{-\infty}^{\infty} \Gamma(j w ; j u) X(j u) d u$ where $X(j w)$ and $Y(j w)$ are the Fourier transforms of $x(t)$ and $y(t)$, respectively. It is also shown that $H(j w ; t)$ satisfies a linear differential equation in $t$ of the same order as $L(p ; t)$. This equation is formally solved for cases where the coefficients of the given equation are either slowly varying functions of time or where they oscillate in the neighborhood of their mean values. (Received October 10, 1949.)

\section{Geometry}

\section{M. H. M. Esser: Self-dual postulates for projective geometry.}

Usually projective geometry is founded on non dual postulates, in which points and lines are fundamental concepts, whereas planes are defined as certain loci of points. Thus the duality between points and planes, which is to be so important later on, is not apparent in the postulates. The author gives a self-dual system of five postulates for $n$-dimensional geometry, in which points and hyperplanes are fundamental, whereas lines and planes of less than $n-1$ dimensions are defined in a self-dual definition. For a space of three dimensions, the number of postulates reduces to four, and they form a system equivalent to the system of postulates found on pages 16 and 24, vol. I, of Projective geometry by $\mathrm{O}$. Veblen and J. Young. The author has simplified certain results by Karl Menger. The details will be published in Duke Math. J. (Received October 13, 1949.) 


\section{LOGIC AND Foundations}

63t. A. R. Schweitzer: Concerning instruments and instrumentalism.

The author describes "instrument" as a means used to attain an end. Discrimination is made between natural and artificial instruments (extension of natural instruments). The hand is a natural instrument for grasping (prehension). From the hand as an instrument of prehension transition is made to discrimination between right and left hands with application, by abstraction, to the foundations of geometry. Any theory dealing with instrument as a leading concept is termed "instrumentalism." In this connection reference is made to Bergson's creative evolution, Whitehead's process and reality, and John Dewey's empiricism as revealed in his Studies in logical theory (Chicago, 1903), Essays in experimental logic (Chicago, 1916), Creative intelligence (New York, 1917), and The quest for certainty (Gifford Lectures, New York, 1929). Reference is also made to the Bridgewater Treatises, Treatise IV: The hand by Sir Charles Bell (London, 1834). (Received October 17, 1849.)

\section{4t. A. R. Schweitzer: On a relation between relativity and quantum theory.}

After a survey of treatises on relativity and quantum theory the author concludes: relativity and quantum theory represent, at least in their present state, divergent lines of development in the evolution of physics; these lines may, however, be so interpreted as to permit motivation by a common concept of energy. (Received October 7, 1949.)

\section{TOPOLOGY}

65t. R. H. Bing: A necessary and sufficient condition that a topological space be metrizable.

A regular topological space $S$ is metrizable if and only if there is a sequence $G_{1}$, $G_{2}, \cdots$ such that (1) $G_{i}$ is a collection of open subsets of $S$ such that the sum of the closures of any subcollection of $G_{i}$ is closed and (2) for each point $p$ and each open set $D$ containing $p$ there is an integer $n(p, D)$ such that an element of $G_{n(p, D)}$ contains $p$ and each element of $G_{n(p, D)}$ containing $p$ is a subset of $D$. An example is given showing that condition (2) above cannot be changed to $\left(2^{\prime}\right)$ for each point $p$ and each open set $D$ containing $p$ there is an integer $n(p, D)$ such that an element of $G_{n(p, D)}$ contains $p$ and is a subset of $D$. The above result is a generalization of the notions of perfect screenability [Bull. Amer. Math. Soc. Abstract 55-7-421] and perfect separability. (Received October 11, 1949.)

66t. S. S. Chern and S. T. Hu: Parallelisability of principal fibre bundles.

A necessary and sufficient condition is given that a principal fibre bundle is parallelisable. The method is based on the examination of the parallelisability of the parts of the bundle over the skeletons of successive dimensions of the base space. If the bundle is $(n-1)$-parallelisable, the $n$-dimensional obstruction elements form a coset $W^{n}(\mathfrak{F}) \in H^{n}\left(B, \pi_{n-1}(G)\right) / P^{n}\left(B, \pi_{n-1}(G)\right)$, where $B$ is the base space, $G$ the reference group, and $P^{n}\left(B, \pi_{n-1}(G)\right)$ the presentable subgroup of the cohomology group. $W^{n}(\mathfrak{F})=0, n=2, \cdots, \operatorname{dim} B$, are then the conditions in question. (Received September 25, 1949.) 


\section{7t. S. S. Chern and Y. F. Sun: The imbedding theorem for fibre bundles.}

The imbedding theorem of Whitney-Steenrod for sphere bundles is generalized to fibre bundles as follows: Let $\mathfrak{F}$ be a fibre bundle over a finite polyhedron $B$ of dimension $n$ as base space. If a principal fibre bundle over $A$ is such that the homotopy groups of its total space vanish up to the dimension $n$ inclusive, the bundle is universal in the sense that the bundles over $B$ are in one-one correspondence with the homotopy classes of maps of $B$ into $A$. This imbedding theorem is also true, if $B$ is a compact metric ANR. It is also extended to the product of fibre bundles, useful for the description of the position of one fibre bundle in another. Discussions are given for the cases that the reference group is a classical group. (Received September 25, 1949.)

\section{R. F. Deniston: On the theory of limits. Preliminary report.}

Application of Zorn's lemma to a partially ordered set, $X$, which is inductive and has the composition property of Moore-Smith (Amer. J. Math. vol, 44, p. 102) assures that $X$ has at least one maximal element, $W_{X}$. A set $X$ having only one maximal element will be called an eventual set and said to converge to the limit, $W_{X}$. If for each point $x$ of a set $E$ is given a nonvoid family of eventual sets $X=\{X\}$ having $x$ as the $W_{X}$ point, a structure is defined by using as an arbitrary neighborhood $N_{X}^{\alpha^{0}}(x)$ the set of all points $y$ for which $y=\alpha_{X}>\alpha_{X}^{0}$ for each $\alpha_{X}^{0}$ and $X \in X$. If all eventual sets on $E$ are isomorphic each to each, the structure given is a uniform structure. Derived eventual sets not originally given may be constructed as follows: For families of points $\left\{a_{z}\right\}$ in $E$ and such that to any $a_{z}$ are related an $X_{1}$ and $X_{2}$ such that $\exists a_{z^{\prime}} \in X_{1}$, $a_{z^{\prime \prime}} \in X_{2}, a_{z^{\prime \prime}}>_{X_{2}} a_{z^{\prime}}>{ }_{X_{1}} a_{z^{\prime}}$. Then the eventual set $Y$ given by $\left\{\cdots>a_{z^{\prime \prime}}>{ }_{X_{2}} a_{z}\right.$ $\left.>_{x_{1}} a_{z^{\prime}}>\cdots\right\}$ has a $W_{Y}$ (by Zorn's lemma), which may correspond to no point in $E$. Regarding a point as given by the collection of eventual sets having it as a $W$ point an extension of the original space is obtained. (Received October 22,1949.)

\section{F. A. Valentine: Arcwise convex sets.}

An arc is said to be convex if it lies in the boundary of its convex hull. A set $S$ is said to be arcwise convex, if each pair of points in $S$ can be joined by a convex $\operatorname{arc}$ in $S$. Theorem 1. Each component of the complement of a bounded closed arcwise convex set in $E_{2}$ (the plane) is arcwise convex. Let $x$ be a point in a set $T \subset E_{2}$ which has the property that each point in $T$ can be joined to $x$ by a convex arc lying in $T$. Designate the set of all such points $x$ by $K$. Theorem 2 . If $T$ is a simply connected continuum in $E_{2}$, then $K$ is a simply connected arcwise convex set. Let $C$ be a continuum in $E_{2}$ such that each pair of points $x$ and $y$ in $C$ lies in a subcontinuum of $C$ contained in one of the halfplanes determined by the line passing through $x$ and $y$. Then Theorem 1 holds for $C$. Theorem 1 is a generalization of a corresponding theorem proved by Horn and Valentine for $L$ sets. (Received October 12,1949.) 